\title{
Association between temporomandibular joint disc position evaluated by magnetic resonance imaging and mandibular condyle inclination evaluated by computed tomography
}

Disc Position and Condylar Inclination

Adriana A. De Stefano ${ }^{1}$ DDS, ME, MScD. PhD candidate Elisabetta Guercio-Monaco ${ }^{1}$ DDS, ME, PhD candidate Adalsa Hernández-Andara, ${ }^{2}$ DDS, MD, $\mathrm{PhD}$ Gabriella Galluccio ${ }^{3}$ DS, DDS

1: Professor. Department of Orthodontics, Faculty of Dentistry, University Central of Venezuela.

2: Researcher. Department of Oral Radiology. Clinic Felix Boada, Caracas, Venezuela

3: Associate Professor - Department of Orthodontics, Faculty of Dentistry. University of Rome Sapienza

\author{
Corresponding author \\ Prof. Adriana A. De Stefano - Universidad Central de Venezuela \\ ID:https://orcid.org/0000-0001-5115-3582 \\ T (+39)33898541892 \\ aadestefano@hotmail.com
}

De Stefano AA, Guercio-Monaco E, Hernández-Andara A, Galluccio G, Guercio-Monaco E, Hernández-Andara A, et al. Association between temporomandibular joint disc position evaluated by magnetic resonance imaging and mandibular condyle inclination evaluated by computed tomography. J Oral Rehabil [Internet]. 2020;(March):joor.12962. Available from: https://onlinelibrary.wiley.com/doi/abs/10.1111/joor.12962 


\section{ABSTRACT}

Temporomandibular joint disc displacement is common in the world's population and could be associated with bone and functional characteristics of the temporomandibular joint.

Objective: to analyze the association between temporomandibular joint disc position evaluated by magnetic resonance imaging (MRI) and the inclination of the mandibular condyle evaluated by computed tomography (CT).

Methods: 170 temporomandibular joints (TMJ) were retrospectively analyzed. The temporomandibular disc position was evaluated by MRI and classified into three types: normal $(\mathrm{N})$, disc displacement with reduction (DDWR) and disc displacement without reduction (DDWoR). The mandibular condyle measurements evaluated by CT included horizontal, sagittal and coronal inclination. ANOVA followed by post hoc Tukey's test was used to evaluate the interaction between condylar inclination and disc position.

.Results: There was an association between disc position and the horizontal and sagittal condylar inclination $(p<0.05)$. There are statistically significant differences in the mean of horizontal and sagittal inclination of the mandibular condyle between the DDWoR and the other disc positions ( $p=0.002$ and $p=0.004)$. Disc position was not statistical associated with coronal inclination of condyle ( $p>0.05)$.

Conclusions: These results indicate that the inclination of the mandibular condyle may be different in TMJ with various disc position. A more medial horizontal inclination and a more posterior sagittal inclination of the mandibular condyle are associated with DDWoR.

Keywords: temporomandibular joint, temporomandibular joint disc, mandibular condyle, magnetic resonance imaging, computed tomography. 


\section{INTRODUCTION}

The temporomandibular joint (TMJ) is part of the craniofacial complex and is made up of a large number of structures and elements, making its operation complex, so that small alterations can cause a wide variety of functional problems ${ }^{1}$. These problems are known as Temporomandibular Disorders (TMD), a generic term covering a group of musculoskeletal and neuromuscular conditions that affect the TMJ, the masticatory muscles and all associated tissues. ${ }^{2,3}$

The TMD are a major health problem, affecting more than $50 \%$ of the world's population at some point in their lives and usually occurs in children, youth and adults of all ages and gender. ${ }^{4,5}$

TMD can be classified into two major categories: temporomandibular joint disorders and masticatory muscle disorders. ${ }^{2,3}$ Among the disorders of the TMJ are the alterations of the intra-articular mobility and the disorders of the disc-condyle complex., ${ }^{2,6}$

Disorders of the disc-condyle complex consist of an abnormal position of the articular disc with respect to the mandibular condyle and the articular eminence. ${ }^{7-9}$

Abnormal disc position or disc displacement has often been diagnosed in patients of diverse populations and can occur even in the growing population and is considered to be a condition that may worsen over time..$^{10,11}$ It has been observed that disc displacement of the TMJ is able to produce pain, ${ }^{10}$ dysfunction and bone changes in the joint surfaces. ${ }^{10,12,13}$

For imaging evaluation of the TMJ, the MRI is considered the gold standard, since it allows obtaining soft tissue images of excellent contrast and has the advantage of being a free ionizing radiation test ${ }^{14-16}$. While computerized tomography $(\mathrm{CT})$ is considered the most 
accurate of traditional radiographic techniques to obtain images of TMJ and evaluate hard tissues. ${ }^{17}$ Recently, conical beam CT (CBCT) has been developed as an alternative to conventional CT for dental and maxillofacial diagnosis, with the benefit of lower ionizing radiation for the patient. ${ }^{18}$ In general, there seems to be no significant differences in diagnostic accuracy for hard tissues of TMJ between CBCT and conventional tomograms. ${ }^{17}$

Several hypotheses have been proposed to explain the etiopathogenesis of disc displacement, including macro and microtrauma in the joint, lack of muscle coordination, ligamentous laxity, changes in TMJ lubrication, postural disorders, stress and depression. ${ }^{19}$ Likewise, the anatomical and functional changes of the osseous structures of the TMJ have been associated with disc displacement. ${ }^{20-25}$

The aim of this investigation was to analyze the association between TMJ disc position evaluated by MRI and the inclination of the mandibular condyle evaluated by CT.

\section{METHODS}

This retrospective cross-sectional study was based on the analysis of MRI and CT obtained from subjects who were referred between 2014 and 2016 to a diagnostic imaging center. The study group was retrospectively selected from the database of institutional images based on the availability of MRI and CT images made with a difference of less than seven days. Diagnostic images involving ionizing radiation were not routinely taken as part of the research protocol. All imaging studies were indicated by the treating physicians of the patients, and the most common reasons for referral were joint noise, jaw locks, limited mouth opening and/or symptoms of ENT (ear, nose and throat). All subjects gave written informed consent about the risks and benefits of conducting these studies, as well as the potential use of the data in future research studies. This study followed the Declaration of Helsinki on 
medical protocol and was endorsed by the Institutional Bioethics Committee of the Faculty of Dentistry of the Central University of Venezuela (CB-017-2017).

Were included in the study patients over 11 years of age, both genders, with CT and MRI of TMJ taken less than 7 days apart. The following exclusion criteria were used: patients with congenital craniofacial malformations or with previous traumas in the area, syndromic patients or with tumors or neoplasms of the head and neck and those who reported systemic inflammatory arthritis or who had undergone maxillofacial surgery or who were under pharmacological treatment.

The final sample was of 170 TMJs, corresponding to 85 patients (62 females and 23 males) aged 11-44 years (mean age $22.6 \pm 10.88$ years).

TMJ MRIs were obtained with an MRI scanner OpticaMR360® GE 1.5-Tesla model (General Electric Medical System, Milwaukee, WI) and with a bilateral $7.5 \mathrm{~cm}$ TMJ antenna. Subjects were placed in supine position, with the condyle in the isocenter of the gantry, the Camper's plane perpendicular to the resonator table. Based on an axial image, $3 \mathrm{~mm}$ thick sections were obtained in the oblique sagittal plane (perpendicular to the anteroposterior longitudinal axis of the mandibular condyle) and oblique coronal plane (parallel to the lateromedial axis of the mandibular condyle). For each TMJ (right and left) the following sequences were obtained: oblique sagittal T1-w, oblique sagittal T2-w, oblique sagittal proton density (PD) and oblique coronal T1-w in closed mouth position and oblique sagittal PD in opened mouth. At the maximum opened mouth position, the subjects were instructed first to open their mouths as far as possible without discomfort. While maintaining a maximum opening, plastic blocks were placed in their mouths during the imaging process.

Tomographic images were obtained with a Multislice Bright Speed ELITE® multidetector (General Electric Medical System, Milwaukee, WI). with each slice being $0.625 \mathrm{~mm}$ 
thickness. The subjects were placed in supine position, with the Camper horizontal plane perpendicular to the scanner table. The gantry had a zero inclination. TMJ images were obtained in the closed mouth. After the scanning was completed, digital imaging and communication in medicine (DICOM) were created with a $1.0 \mathrm{~mm}$ slice thickness. The acquired 2D CT DICOM data were then input into a personal computer. The image evaluation was performed using the AWCOD software of the Advantage Workstation AWCID 4.6-2010 workstation (GE Healthcare).

The images were interpreted by three trained researchers, one experienced radiologist and two orthodontists with experience. Images that were not clear enough were excluded.

The position of the articular disc was evaluated in TMJ MRIs in the maximum intercuspation and in the full open-mouth position. The combination of both positions constituted the position of the articular disc.

The evaluation of the position of the disc position in closed-mouth was performed in the sagittal and coronal planes:Normal disc position: in the sagittal plane, relative to the superior aspect of the condyle, the border between the low signal of the disc and the high signal of the retrodiscal tissue is located between the 11:30 and 12:30 clock positions and the intermediate zone is located between the anterior-superior aspect of the condyle and the posterior-inferior aspect of the articular eminence; in the oblique coronal plane, the disc is centered between the condyle and eminence in the medial, central, and lateral parts. Disc displacement: when the low signal of the disc and the high signal of the retrodiscal tissue are located anterior to the $11: 30$ clock position relative to the superior aspect of the condyle and, the intermediate zone of the disc is located anterior to the condyle or the disc is not centered between the condyle and eminence in either the medial or the lateral parts in the axially corrected coronal plane..$^{3,8,9,26}$ 
The evaluation of the disc position in open-mouth, may present the following positions: normal disc position when the intermediate zone of the disc was between the articular eminence and the condyle articular surface or persistent disc displacement when the intermediate zone is located anterior to the condylar head. $3,27-29$

The TMJ disc position was obtained from the combination of the closed-mouth and openmouth positions and a final diagnosis was formulated for each joint. It was divided into three categories according to the following criteria: ${ }^{3}$ (Figure 1)

1. Normal (N): Disc location is normal on closed- and open-mouth images.

2. Disc displacement with reduction (DDWR): Disc location is displaced on closedmouth images but normal in open-mouth images

3. Disc displacement without reduction (DDWoR): Disc location is displaced on closed-mouth and open-mouth images.

The following inclination of the mandibular condyle was obtained in grade by CT in a closed mouth: the horizontal condylar inclination angle formed between the long axis of the condyle and sagittal midline in the axial plane. Sagittal midline corresponds to the line passing through the basion and the crista galli apophysis ${ }^{20,30-32}$ (Figure 2). Sagittal condylar inclination: Angle formed between the axis of the condyle and the tangent of the mandibular branch in the sagittal plane. The axis of the mandibular condyle corresponds to the line joining the uppermost point of the mandibular condyle to the midpoint of a line perpendicular to the tangent of the branch that intercepts the outermost point of the mandibular condyle and the anterior contour thereof ${ }^{31,33}$ (Figure 3). Coronal condylar inclination: Angle formed by the intersection of the line connecting the center (CC) and laterosuperior point (PLS) of the mandibular condyle with the midsagittal plane in the coronal plane. CC: condyle center located in the axial plane at the intersection of the largest lateromedial diameter with the largest anteroposterior diameter. PLS: intersection of a 
tangent to the condyle's upper edge with a tangent to the condyle's side edge in the coronal section where the center of the mandibular condyle is located ${ }^{32}$ (Figure 4).

A pilot study was conducted to determine the reliability of the data collection procedure. It randomly included 15 subjects (30 TMJ). A 2-week interval was using for the intra-rater reliability. To test the condylar inclination reliability, intra-class correlation coefficients (ICC) were calculated for each examiner. The reliability of the horizontal condyle inclination was considered as excellent (ICC $=0.954,0.923$ and 0.918$)$ and that of sagittal and coronal condyle inclination as good $(\mathrm{ICC}=0.728,0.786$ and 0.792 and $\mathrm{ICC}=0.726,0.764$ and 0.739 respectively). The intra-examiner reliability of the disc position was determined using Cohen's Kappa. The Kappa value for each examiner was $0.85,0.87$ and 0.89 respectively, which were considered as very good. Inter-rater reliability for quantitative data was assessed with inter-class correlation coefficient (ICC) and for qualitative data with Cohen's Kappa. The reliability of horizontal condyle inclination was considered as very good between examiners (ICC $=0.832)$, and that of sagittal and coronal condyle inclination as good $(\mathrm{ICC}=0.711$ and 0.724 respectively). The reliability of disc position between examiners was $k=0.89$, considered as very good. Consequently, all measurements were considered suitable for usage in the study.

The data was performed using SPSS software, version 21.0 (SPSS Institute Inc., Chicago, USA). A descriptive analysis was performed through statistical frequencies for qualitative variables, for continuous variables parameters were expressed as mean \pm SD (standard deviation). ANOVA followed by post hoc Tukey's test were used to assess the statistical association between TMJ disc position and the inclination of the mandibular condyle. Statistical significance was defined as $p<0.05$. 


\section{RESULTS}

One hundred seventy TMJs were analyzed of 85 subjects ( 62 females and 23 males, mean age $22.6 \pm 10.88$ years). The frequency of the observed TMJ disc position was normal position (N) in $20 \mathrm{TMJ}(11.8 \%)$, disc displacement with reduction (DDWR) in $101 \mathrm{TMJ}$ (59.4\%) and disc displacement without reduction (DDWoR) in 49 TMJ (28.8\%). A statistical association between disc displacements and gender $(p=0.002)$ was observed, with more frequent disc displacements in females.

For the associations between the TMJ disc position and mandibular condyle inclinations, it was decided to use the complete sample, since no differences were observed in the associations under study by age groups separated: growing patients (11-18 years of age) and adults ( $>18$ years old).

There was no statistical difference between TMJ disc position and coronal inclination of mandibular condyle $(p=0.285)$. There was a statistically significant difference between the TMJ disc position and the horizontal and sagittal inclination of mandibular condyle ( $p=0.002$ and $p=0.004$ respectively) (Table 1 ). The post hoc test revealed that there are statistically significant differences in the horizontal inclination of the mandibular condyle between the DDWoR and the other disc positions ( $p=0.002$ ). When observing the means of the horizontal angle of the mandibular condyle in the different disc positions, it is evident that DDWoR presents the lowest mean $\left(57.74^{\circ} \pm 9.09^{\circ}\right)$ with respect to the rest of the positions. In sagittal inclination, multiple comparisons also indicate a significant difference in the mean of the DDWoR with the other two groups $(p=0.004)$, with a greater mean of this angle observed in cases with disc displacement without reduction $\left(8.05^{\circ} \pm 4.61^{\circ}\right)$ 


\section{DISCUSSION}

The results obtained in this study are based on the analysis of MRI and CT, methods reported as gold standards for the evaluation of the structures of the TMJ. ${ }^{15,34}$ However, CBCT is suggested as an alternative of lower radiation dose for patients ${ }^{17,35}$. The sample of this study was obtained by retrospective recruitment of images from a Diagnostic Center for imaging based on the availability of CT and MRI. ${ }^{17}$

It was not possible to perform the clinical evaluation of the patients, due to the retrospective collection of the images. The clinical data found in the reports of the referring physicians were incomplete and therefore were not incorporated in the analysis.

As in other studies, disc displacements showed a significantly higher prevalence in females. ${ }^{8,9,36,37}$ However, studies such as Foucart et al ${ }^{38}$., Larheim et al. ${ }^{26}$ and CrusoéRebello et al. ${ }^{39}$ do not reflect gender difference in disc displacement prevalence. The causes of the increased prevalence of disc displacement in females are not entirely clear, however, some theories have been proposed. The first one states that this difference is related to a greater demand for treatment of women and not with increased susceptibility to degenerative diseases of TMJ. ${ }^{40}$ Another suggests that the difference is due to a change in collagen metabolism associated with joint laxity of genetic origin. ${ }^{36}$ Some studies also suggest that estradiol, the female sex hormone, induces proinflammatory cytokines, aggravating the inflammation of the TMJ. ${ }^{36,37}$

The DDwR was the most prevalent disc position (59.4\%), as reported by other studies. ${ }^{21,41,42}$ The disc reduction capacity has been related to anatomical characteristics of the mandibular condyle and mandibular fossa. ${ }^{23,24}$ When the displacement is complete, the condyle tends to move closer to the articular eminence, pressing retrodiscal tissue, minimizing the possibility of reduction. ${ }^{8}$ Adapting the articular disc between the osseous components of the 
TMJ either at rest as in the opening and closing movement of the mouth is partly guaranteed by its morphology and by ligaments that support it. ${ }^{43,44}$ However, this adaptation can be influenced by some parameters of joint bone morphology such as the inclination of the mandibular condyle. ${ }^{20,39,45}$

In this investigation, a positive association between the horizontal condylar inclination and articular disc position was found. Busato et al. ${ }^{20}$ and Raustia et al. ${ }^{45}$ consider that horizontal condylar inclination is a variable dividing healthy individuals from patients with disc incoordination. Williamson et al. ${ }^{45}$ evaluated horizontal condylar inclination, between the axial condylar axis and the sagittal midline, finding an association between this inclination and the joint condition, as reported in the present study.

The means of the horizontal angle of the mandibular condyle showed that the DDWoR has the lowest average $\left(57.74^{\circ}\right)$, the condyle is more medially rotated. Crusoé-Rebello et al. ${ }^{39}$, Kurita et al. ${ }^{46}$ and Pregatz et al. ${ }^{47}$ assessed the horizontal inclination in MRI by relating it to a coronal plane, so that the increase in the angle represents condyles turned towards medial, while reduced angles represent condyles turned laterally or more horizontally. The results obtained by Crusoé-Rebello et al. ${ }^{39}$ showed that the average value of the horizontal condylar angles was higher in individuals with a positive diagnosis of disc displacement. Similarly, Kurita et al..$^{46}$ found that higher angles, that is, rotated medially, were associated with the displacement without reduction, as reported in the present study.

In coincidence Westesson et al. ${ }^{48}$ reported that the horizontal condylar angle becomes increasingly larger, the condyle rotated towards the medial, with more advanced changes related to disc displacement $(\mathrm{DDW}$ R $>$ DDWR $>N$ ). These authors report that there are several possible explanations for the increased horizontal angle in joints with disc displacement. Joints with a greater condylar angle (rotated medially) may have a greater 
tendency to disc displacement due to the greater tendency of lateral ligament stretch during mouth opening movements. Another explanation could be that the remodeling associated with disc displacement may result in a greater condylar angle. Reinforcing this explanation, Sülün et al. ${ }^{49}$ propose that the difference between the horizontal condylar inclination in patients with disc displacement and asymptomatic volunteers may be the result of remodeling or degeneration caused by the disorder.

In contrast, Pregarz et al. ${ }^{47}$ reported results in which more horizontal condyles were associated with disc displacement without reduction. While Amorin et al. ${ }^{42}$ found no association between the horizontal condylar inclination and disc displacement.

The sagittal condylar inclination was associated with the position of the articular disc in the sample studied, showing that the DDWoR occur in more distally inclined condyles as also reported by Ahn et al. ${ }^{31}$ who observed that the small and distally inclined condyles were evident in subjects with DDWoR. This might be related to hard tissue remodeling and bone degenerative changes in the advanced stages of disc displacement in the TMJ.

Regarding the coronal condylar angle, when comparing the means in the different positions of the disc, no statistical association was evident, like Almǎşan et al. ${ }^{50}$ However, it can be observed that in the coronal displacements, the coronal angle is smaller (rotated medially) than in the TMJ discs in normal position, as shown by Raustia et al ${ }^{45}$ who found differences in this inclination between patients with temporomandibular dysfunction and the control group.

Various investigations have been carried out analyzing the association between the mandibular condyle inclination, especially the horizontal inclination, and the position of the articular disc and the results remain controversial. ${ }^{39,42,47,49}$ In addition, due to the cross- 
sectional design of the studies, including the present, it is not possible to establish a cause and effect relationship between the angles of the condyle and the positions of the disc.

\section{CONCLUSIONS}

An association between TMJ disc position and horizontal and sagittal mandibular condyle inclination was observed. However, no association between disc position and coronal inclination of mandibular condyle. These results indicate that the inclination of the mandibular condyle may be different in TMJ with various disc position. A more medial horizontal inclination and a more posterior sagittal inclination of the mandibular condyle are associated with DDWoR.

Additional studies are required to further understand the complex nature of the TMJ disc displacement and its association with join morphology.

\section{Disclosure statement}

No potential conflict of interest was reported by the authors.

\section{REFERENCES}

1. Prendes Rodríguez AM, Martínez Brito I, Faget Mora M. La disfunción temporomandibular y su relación con algunos factores de riesgo en niños de 7 a 11 años: Los Arabos, Matanzas. Rev medica electron. 2014;36(1):15-24.

2. de Leeuw R. American Academy of Orofacial Pain. Oro Facial Pain: Guidelines for Assessment, Diagnosis and Manegement. Quintessence. 2008. 316 p.

3. Schiffman E, Ohrbach R, Truelove E, Look J, Anderson G, Goulet J-P, et al. Diagnostic Criteria for Temporomandibular Disorders (DC/TMD) for Clinical and Research Applications: Recommendations of the International RDC/TMD Consortium Network $^{*}$ and Orofacial Pain Special Interest Group†. J oral facial pain headache. 2014;28(1):6-27. 
4. Da Silva CG, Pachêco-Pereira C, Porporatti AL, Savi MG, Peres MA, Flores-Mir C, et al. Prevalence of clinical signs of intra-articular temporomandibular disorders in children and adolescents A systematic review and meta-analysis. J Am Dent Assoc. 2015;147(1):10-18e8.

5. Committee O. Clinical guideline on acquired temporomandibular disorders in infants, children, and adolescents. Am Acad Pediatr Dent. 2015;37(6):272-8.

6. Peck Christopher, Goulet Jean-Paul, Lobbezoo F, Schiffman E, Per A, Anderson G. Expanding the Taxonomy of the Diagnostic Criteria for Temporomandibular Disorders (DC/TMD). J Oral Rehabil. 2014;41(1):2-23.

7. Katzberg RW, Westesson P-L, Tallents RH, Anderson R, Kurita K, Manzione J V, et al. Temporomandibular Rotational and Joint: Sideways MR Assessment Disk Displacements. Radiology. 1988;169:741-8.

8. Milano V, Desiate A, Bellino R, Garofalo $T$. Magnetic resonance imaging of temporomandibular disorders. Dentomaxillofacial Radiol. 2000;29:352-61.

9. Tasaki MM, Westesson PL, Isberg a M, Ren YF, Tallents RH. Classification and prevalence of temporomandibular joint disk displacement in patients and symptomfree volunteers. Am J Orthod Dentofac Orthop. 1996;109(3):249-62.

10. De Melo DP, Sousa Melo SL, De Andrade Freitas Oliveira LS, Ramos-Perez FMDM, Campos PSF. Evaluation of temporomandibular joint disk displacement and its correlation with pain and osseous abnormalities in symptomatic young patients with magnetic resonance imaging. Oral Surg Oral Med Oral Pathol Oral Radiol. 2015;119(1):107-12.

11. Marpaung C, Lobbezoo F, van Selms MKA. Temporomandibular Disorders among Dutch Adolescents: Prevalence and Biological, Psychological, and Social Risk Indicators. Pain Res Manag. 2018:1-9.

12. Dolwick MF. Intra Articular Disc displacement Part I: Its questionable role in Temporomandibular Joint Pathology. J Oral Maxillofac Surg. 1995;53:1069-72.

13. Gil C, Santos KCP, Dutra MEP, Kodaira SK, Oliveira JX. MRI analysis of the relationship between bone changes in the temporomandibular joint and articular disc 
position in symptomatic patients. Dentomaxillofacial Radiol. 2012;41(5):367-72.

14. Alarabawy RA, El Ahwal HM, El Sergany MAES, Mehrez WW. Magnetic resonance imaging evaluation of temporo-mandibular joint disorders, criterial analysis and significance in comparison with arthroscopy. Egypt $\mathrm{J}$ Radiol Nucl Med. 2016;47(2):467-75.

15. Al-Saleh MAQ, Alsufyani NA, Saltaji $\mathrm{H}$, Jaremko JL, Major PW. MRI and CBCT image registration of temporomandibular joint: A systematic review. J Otolaryngol - Head Neck Surg. 2016;45(1):30.

16. Talmaceanu D, Lenghel LM, Bolog N, Hedesiu M, Buduru S, Rotar H, et al. Imaging modalities for temporomandibular joint disorders: An update. Dent Med. 2018;91(3):280-7.

17. Hintze $\mathrm{H}$, Wiese $\mathrm{M}$, Wenzel $\mathrm{A}$. Cone beam $\mathrm{CT}$ and conventional tomography for the detection of morphological temporomandibular joint changes. Dentomaxillofacial Radiol. 2007;36(4):192-7.

18. Caruso S, Storti E, Nota A, Ehsani S, Gatto R. Temporomandibular joint anatomy assessed by CBCT images. Vol. 2017, BioMed Research International. 2017.

19. Manfredini D. Etiopathogenesis of disk displacement of the temporomandibular joint: a review of the mechanisms. Indian J Dent Res. 2009;20(2):212-21.

20. Busato A, Vismara V, Bertele L, Zollino I, Fanali S, Trapella G, et al. Does Tmj Morphology Determine Disk Incoordination ? Eur J Inflamm. 2011;9(1(S)):19-23.

21. de Farias JFG, Melo SLS, Bento PM, Oliveira LSAF, Campos PSF, de Melo DP. Correlation between temporomandibular joint morphology and disc displacement by MRI. Dentomaxillofacial Radiol. 2015;44(7):1-7.

22. Kinniburgh RD, Major PW, Nebbe B, West K, Glover KE. Osseous Morphology and Spatial Relationships of the Temporomandibular Joint: Comparisons of Normal and Anterior Disc Positions. Angle Orthod. 2000;70(1):70-80.

23. Pullinger AG, Seligman DA, John MT, Harkins S. Multifactorial modeling of temporomandibular anatomic and orthopedic relationships in normal versus 
undifferentiated disk displacement joints. J Prosthet Dent. 2002;87:289-97.

24. Seligman D a, Pullinger AG. Improved interaction models of temporomandibular joint anatomic relationships in asymptomatic subjects and patients with disc displacement with or without reduction. J Orofac Pain. 2004;18(3):192-202.

25. Guercio Monaco E, De Stefano AA, Hernandez-Andara A, Galluccio G. Correlation between condylar size on CT and position of the articular disc on MRI of the temporomandibular joint. CRANIO® J Craniomandib Sleep Pract. 2019;00(00).

26. Larheim $\mathrm{T}$ a, Westesson $\mathrm{P}$, Sano $\mathrm{T}$. Temporomandibular joint disk displacement: Comparison in asymptomatic volunteers and patients. Radiology. $2001 ; 218(2): 428-$ 32.

27. Eberhard D, Bantleon HP, Steger W. The efficacy of anterior repositioning splint therapy studied by magnetic resonance imaging. Eur J Orthod. 2002;24(4):343-52.

28. Choi Y-S, Asaumi J-I, Hisatomi M, Unetsubo T, Yanagi Y, Matsuzaki H, et al. Analysis of magnetic resonance images of disk positions and deformities in 1,265 patients with temporomandibular disorder. Open Dent J. 2009;3(FEBRUARY 2009):1-20.

29. Kalaykova S, Naeije M, Huddleston Slater JJR, Lobbezoo F. Is condylar position a predictor for functional signs of TMJ hypermobility? J Oral Rehabil. 2006;33(5):34955 .

30. Rodrigues AF, Fraga MR, Vitral RWF. Computed tomography evaluation of the temporomandibular joint in Class II Division 1 and Class III malocclusion patients: Condylar symmetry and condyle-fossa relationship. Am J Orthod Dentofac Orthop. 2009;136(2):199-206.

31. Ahn SJ, Kim T-W, Lee D-Y, Nahm D-S. Evaluation of internal derangement of the temporomandibular joint by panoramic radiographs compared with magnetic resonance imaging. Am J Orthod Dentofac Orthop. 2006;129:479-85.

32. Melgaço CA, Neto JC, Jurach EM, Nojima MDCG, Nojima LI. Immediate changes in condylar position after rapid maxillary expansion. Am J Orthod Dentofac Orthop. 2014;145(6):771-9. 
33. Goyal A, Kamble RH, Shrivastav S, Sharma N. Three dimensional evaluation of condylar head inclination with respect to ramus among post pubertal Class II patterns. Int J Cur Res Rev. 2015;7(9):20-7.

34. Barchetti F, Stagnitti A, Glorioso M, Al Ansari N, Barchetti G, Pranno N, et al. Static and dynamic MR imaging in the evaluation of temporomandibular disorders. Eur Rev Med Pharmacol Sci. 2014 Oct;18(20):2983-7.

35. Larheim TA, Abrahamsson AK, Kristensen M, Arvidsson LZ. Temporomandibular joint diagnostics using CBCT. Dentomaxillofacial Radiol. 2015;44(1):1-12.

36. Bagis B, Ayaz EA, Turgut S, Durkan R, Özcan M. Gender Difference in Prevalence of Signs and Symptoms of Temporoman- dibular Joint Disorders : A Retrospective Study on 243 Consecutive Patients. Int J Med Sci. 2012;9:539-44.

37. Witzel AL, Costa JE, Bolzan MC, Carli ML De, Nunes TB, Di Matteo RC, et al. Correlation between gender, temporomandibular joint disc position and clinical findings in patients with temporomandibular disorder. MedicalExpress. 2015;2(4):15.

38. Foucart JM, Carpentier P, Pajoni D, Marguelles-Bonnet R, Pharaboz C. MR of 732 TMJS: Anterior, rotational, partial and sideways disc displacements. Eur J Radiol. 1998;28(1):86-94.

39. Crusoé-rebello IMR, Sérgio P, Campos F, Regina I, Rubira F. Evaluation of the relation between the horizontal condylar angle and the internal derangement of the TMJ - a magnetic resonance imaging study Avaliação da relação entre o ângulo horizontal do côndilo e o. Pesqui Odontol Bras. 2003;17(2):176-82.

40. Isberg a, Hägglund M, Paesani D. The effect of age and gender on the onset of symptomatic temporomandibular joint disk displacement. Oral Surg Oral Med Oral Pathol Oral Radiol Endod. 1998;85(3):252-7.

41. Vieira-Queiroz I, Gomes Torres MG, de Oliveira-Santos C, Flores Campos PS, Crusoé-Rebello IM. Biometric parameters of the temporomandibular joint and association with disc displacement and pain: a magnetic resonance imaging study. Int J Oral Maxillofac Surg. 2013 Jun 1;42(6):765-70. 
42. Amorim MY, Alves MGO, Almeida JD, Montesinos GAP, Costa ALF, Lopes SLP d. C. Inclination of the condylar long axis is not related to temporomandibular disc displacement. J Investig Clin Dent. 2018;10(1):1-6.

43. Fuentes R, Ottone NE, Bucchi C, Cantín. Analysis of Terms Used in the Literature to Refer to Temporomandibular Joint Capsule and Joint Ligaments Ramón [Análisis de los términos utilizados en la literatura científica para referirse a la Cápsula Articular y Ligamentos Articulares de la Articulaci. Int J Morphol. 2016;34(1):342-50.

44. Fuentes R, Cantin M, Ottone N, Bucchi C. Characterization of Bone Components of the Temporomandibular Joint. A Literature Review. Fuentes [Caracterización de los Componentes Óseos de la Articulación] Temporomandibular. Una Revisión de la Literatura. Int J Morphol. 2015;33(4):1569-76.

45. Raustia AM, Pyhtinen J. Morphology of the condyles and mandibular fossa as seen by computed tomography. J Prosthet Dent. 1990;63(1):77-82.

46. Kurita H, Ohtsuka A, Kobayashi H, Kurashina K. Relationship between increased horizontal condylar angle and resorption of the posterosuperior region of the lateral pole of the mandibular condyle in temporomandibular joint internal derangement. Dentomaxillofacial Radiol. 2003;32(1):26-9.

47. Pregarz M, Bodin C. MRI evaluation of TMJ condylar angulations. European Society of Radiology; 2010. p. 1-10.

48. Westesson P, Bifano J, Tallents R, Hatala MP. Increased horizontal angle of the mandibular condyle in abnormal temporomandibular joints. Oral Surg Oral Med Oral Pathol Oral Radiol Endod. 1991;72(3):359-63.

49. Sülün T, Akkayan B, Duc JMP, Rammelsberg P, Tuncer N, Gernet W. Axial Condyle Morphology and Horizontal Condylar Angle in Patients with Internal Derangement Compared to Asymptomatic Volunteers. Cranio. 2001;19(4):237-45.

50. Almăşan OC, Hedeşiu M, Băciuţ G, Leucuţa DC, Băciuţ M. Disk and joint morphology variations on coronal and sagittal MRI in temporomandibular joint disorders. Clin Oral Investig. 2013;17(4):1243-50. 
Table 1. Association between disc position and condylar inclination

\begin{tabular}{llrrrrr}
\hline & Disc Position & N (\%) & \multicolumn{1}{l}{ Mean } & SD & p-value \\
& & & & & & \\
\hline \multirow{3}{*}{ Horizontal Inclination } & Normal & 12 & 66.46 & 8.91 & \\
& DDWR & 59 & 67.31 & 10.04 & $0.002^{*}$ \\
& DDWoR & 29 & 57.74 & 9.09 & \\
\hline \multirow{3}{*}{ Coronal Inclination } & Normal & 12 & 52.01 & 6.63 & \\
& DDWR & 59 & 49.20 & 8.15 & 0.326 \\
& DDWoR & 29 & 49.98 & 7.24 & \\
\hline \multirow{3}{*}{ Sagittal Inclination } & Normal & 12 & 4.63 & 7.46 & \\
& DDWR & 59 & 4.57 & 5.94 & $0.004^{*}$ \\
& DDWoR & 29 & 8.05 & 4.61 &
\end{tabular}

$\mathrm{N}(\%)$ : absolute frequency; SD: Standard deviation; DDWR: Disc displacement with reduction; DDWoR: Disc displacement without reduction. *ANOVA test $p$-value $<0.05$ indicated statistical differences 


\section{List of Figure}

Figure 1. Disc Position: Normal, disc displacement with reduction (DDWR), disc displacement without reduction (DDWoR)

Figure 2. Horizontal inclination of the mandibular condyle

Figure 3. Sagittal condylar inclination

Figure 4. Coronal condylar inclination 